\title{
Avaliação Hemodinâmica e Evolução Clínica de Crianças Portadoras de Cardiomiopatia Dilatada Grave Candidatas a Transplante Cardíaco
}

\author{
Estela Azeka, Miguel Barbero Marcial, Paulo Roberto Camargo, Luiz Kajita, \\ Ana Cristina Aliman, José Otóvio C. Auler, Edmar Atik, Munir Ebaid
}

São Paulo, SP

\begin{abstract}
Objetivo - Estudar o perfil dos parâmetros hemodinâmicos e a evolução clínica de crianças candidatas a transplante cardíaco, portadoras de cardiomiopatia grave.

Métodos - Foram 24 crianças, com idade entre 4 meses e 10 anos e 8 meses (média de 3,7 2,5 anos), no periodo de fevereiro/92 a maio/96, submetidas a estudo hemodinâmico e medidos os seguintes parâmetros: débito cardíaco, pressão média de artéria pulmonar (PMAP) e pressão capilar pulmonar. Foram calculados o indice de resistência vascular pulmonar (IRVP) e gradiente de pressão transpulmonar (GPT).

Resultados - Do ponto de vista evolutivo, $10(41,6 \%)$ crianças foram transplantadas (grupo A), 5 (20,8\%) aguardam o transplante (grupo B) e $9(37,6 \%$ ) faleceram (grupo C). Observou-se que a média das idades dos pacientes do grupo B foi significativamente menor que do grupo C. Dos dados hemodinâmicos, a PMAP, GTP e IRVP apresentaram médias significativamente menores no grupo $A$ em relação ao grupo $C$.

Conclusão - O perfil hemodinâmico de crianças candidatas ao transplante cardiaco mostrou-se compatível ao quadro clínico de insuficiência cardiaca grave. $A$ idade foi o único fator que diferenciou o grupo $B$ e $C$ $(p=0,036)$. OIRVP, PMAP e o GTP foram fatores que diferenciaram de modo significativo o grupo A e o grupo $C$ ( $p=0,010 ; p=0,044$ e $p=0,023$, respectivamente). Quanto maior a idade no momento da indicação do transplante na criança, pior foi seu prognóstico.
\end{abstract}

Palavras-chave: criança, cardiomiopatia dilatada, transplante cardíaco

\section{Hemodynamic Evaluation and Clinical Outcome of Children Candidates for Heart Transplantation with Severe Dilated Cardiomyopathy}

Purpose - To evaluate hemodynamic parameters and clinical outcome of children with severe cardiomyopathy who are candidates for heart transplantation.

Methods - Twenty four children aged from 4 months to 10 years and 8 months (mean $3.7 \pm 2.5$ years) from February 1992 to May 1996, were submitted to hemodynamic study and the following parameters were measured: cardiac output, mean pulmonary artery pressure (MPAP) and capillary wedge pressure. The pulmonary vascular resistance index (PVRI) and transpulmonary pressure gradient (TGP) were calculated.

Results - Ten (41.6\%) children were transplanted (group A), 5 (20.8\%) are still waiting heart transplantation (group B) and 9 (37.6\%) died (group C). The mean age of the $B$ and $C$ group were significantly different. The PVRI, MPAP and TPG were significant lower in group $A$ than group $C(p=0.01 ; p=0.044$ and $p=0.023$ respectively).

Conclusion - The profile of the hemodynamic parameters of children with severe dilated cardiomyopathy was compatible with the clinical findings. The age was the only difference comparing groups $B$ and C. PVRI, MPAP and $T P G$ were significant factors between group $A$ and $C$. The older the patient, the worse was the prognosis.

Key-words: child, dilated cardiomyopathy, heart transplantation
Instituto do Coração do Hospital das Clínicas - FMUSP Correspondência: Miguel Barbero Marcial - Incor - Av. Dr. Enéas C. Aguiar, 44 - $2^{\circ}$ - Divisão de Cirurgia - 05403-000 - São Paulo, SP Recebido para publicação em 30/3/98 Aceito em 5/8/98
Crianças portadoras de cardiomiopatia dilatada com disfunção hemodinâmica são submetidas à investigação diagnóstica e terapêutica convencional e/ou imunossupressora quando portadoras de miocardite ${ }^{1}$. 
A experiência constatou que um grupo apresentou evolução clínica desfavorável com mortalidade elevada. Essas crianças caracterizam-se por acentuação progressiva da insuficiência cardíaca (IC), fração de ejeção de ventrículo esquerdo (FEVE) através da ventriculografia radioisótopica demonstrando disfunção grave, cintilografia com gálio 67 negativa e/ou biópsia endomiocárdica sem evidência de processo inflamatório ${ }^{2-6}$. O transplante cardíaco constitui a opção terapêutica a esse grupo de crianças que até então tinha perspectivas limitadas de sobrevida ${ }^{7-9}$.

A indicação do procedimento deve incluir estudo rigoroso dos parâmetros hemodinâmicos e o cálculo do índice de resistência vascular pulmonar (IRVP) que deve ser menor que 6 unidades Wood ${ }^{10-14}$.

A avaliação dos parâmetros hemodinâmicos bem como a análise evolutiva são fatores decisivos na indicação do tratamento cirúrgico e no tipo de transplante, bem como o risco de desenvolvimento de disfunção ventricular direita peri-operatória e o prognóstico a longo prazo ${ }^{10}$.

O objetivo principal do estudo foi o de avaliar hemodinamicamente as crianças selecionadas clinicamente para o transplante e obter, assim, dados indispensáveis para a indicação.

\section{Métodos}

Foram estudadas 24 crianças portadoras de cardiomiopatia dilatada com idades de 4 meses a 10 anos e 8 meses (média de 3,7 72,5 anos) no Instituto do Coração HCFMUSP em IC refratária (classe funcional (CF) IV,NYHA)e submetidas a exames de investigação da cardiopatia com radiografia de tórax, eletrocardiograma (ECG), ecoDopplercardiograma, cintilografia com gálio 67 e/ou biópsia endomiocárdica, ventriculografia radioisotópica e cateterismo cardíaco direito. O método de termodiluição (Swan-Ganz) foi utilizado no cateterismo cardíaco para medir o débito cardíaco, a pressão média de artéria pulmonar (PMAP), a pressão capilar pulmonar e calcular através das medidas obtidas o IRVP e gradiente de pressão transpulmonar (GPT).

As crianças foram acompanhadas do ponto de vista cardiológico, sendo divididas em três grupos segundo sua evolução: o grupo (A) que recebeu o transplante, o grupo (B) que aguarda o transplante e o grupo (C) que faleceram enquanto aguardavam o transplante. Apesar do grupo A ter tido sua evolução natural interrompida pela oportunidade cirúrgica, surgida quando os doadores foram encontrados, achamos de interesse incluí-lo no estudo comparativo com os outros dois grupos, uma vez que a análise hemodinâmica global dos 24 pacientes permitirá estabelecer um perfil hemodinâmico deste tipo de cardiopatia, podendo ser de utilidade no momento de seleção dos pacientes candidatos a transplante cardíaco.

Os três grupos foram analisados e comparados segundo a idade, fração de encurtamento pelo ecodopplercardiograma, FE pela ventriculografia radioisotópica, PMAP, pressão capilar pulmonar, IRVPe GPT.

A análise estatística consistiu de método paramétrico com análise de variância (teste de Student-Newman-Keuls) e não paramétrico: Kruskal-Wallis (teste de Dunn).

\section{Resultados}

As 24 crianças apresentaram ao ecodopplercardiograma fração de encurtamento de 5\% a 25\% (média de $14,1 \pm 3,9 \%$ ). A ventriculografia radioisotópica foi de $8 \%$ a $26 \%$ de FEVE (média de 16,9 $\pm 4,6 \%$ ) e a cintilografia e/ou biópsia endomiocárdica com resultado negativo.

Do ponto de vista evolutivo, $10(41,6 \%)$ crianças foram transplantadas, $5(21,7 \%)$ aguardam o transplante e 9 $(37,6 \%)$ faleceram. O tempo total de evolução variou de 0,5 a 12 meses (média de 3,1 meses). O tempo de evolução do grupo A variou de 0,5 a 4 meses (média de 2,7 meses), do grupo $\mathrm{B}$ de 2 a 12 meses (média de 6,4 meses) e do grupo $\mathrm{C}$ de 0,6 a 4 meses (média de 1,8 meses). Todos os pacientes do grupo C faleceram por choque cardiogênico, internados em Unidade de Terapia Intensiva do Incor.

O número de internações prévias do grupo A variou de duas a sete internações (média de 4,4 internações), no grupo $\mathrm{B}$ de zero a três internações (média de 1,6 internações) e no grupo $\mathrm{C}$ de uma a quatro internações (média de duas internações), houve diferença significativa do número de internações entre o grupo $A$ em relação ao grupo $B$ e grupo $C,(p=0,005)$.

Observou-se que no grupo $\mathrm{A}$ apenas um paciente utilizava dobutamina, na dose de $8 \mu \mathrm{g} / \mathrm{kg} / \mathrm{min}$ no momento do transplante; no grupo B não observamos pacientes com drogas vasoativas na última avaliação realizada; no grupo $C$, os pacientes receberam dobutamina na dose de 10 a $25 \mu \mathrm{g} / \mathrm{kg} /$

\begin{tabular}{|c|c|c|c|}
\hline \multicolumn{4}{|c|}{ Tabela I - Idade (meses) } \\
\hline \multicolumn{2}{|c|}{ Grupo A } & \multirow{2}{*}{$\frac{\text { Grupo B }}{27}$} & \multirow{2}{*}{$\begin{array}{c}\text { Grupo C } \\
36\end{array}$} \\
\hline & 59 & & \\
\hline & 4 & 29 & 36 \\
\hline & 46 & 7 & 66 \\
\hline & 20 & 38 & 129 \\
\hline & 31 & 37 & 101 \\
\hline & 61 & & 24 \\
\hline & 70 & & 68 \\
\hline & 24 & & 84 \\
\hline & 12 & & 33 \\
\hline & 30 & & \\
\hline Média & 35,70 & 27,60 & 64,11 \\
\hline DP & 22,29 & 12,48 & 35,61 \\
\hline
\end{tabular}

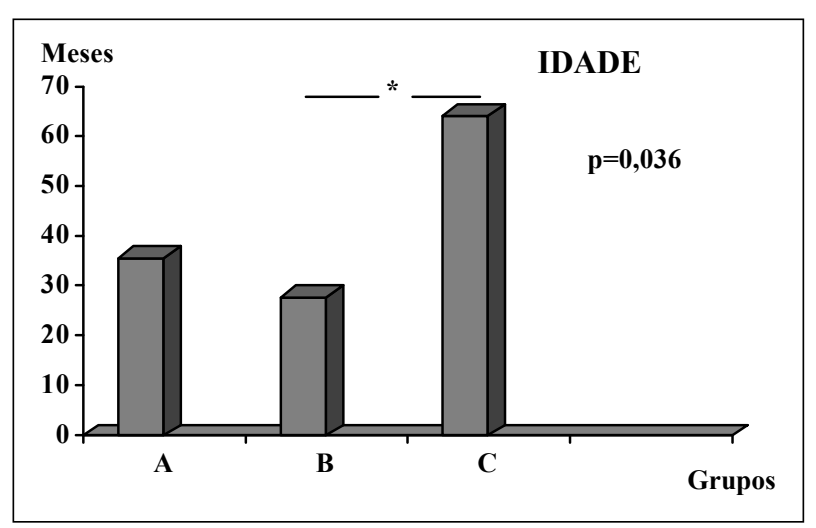

Fig. 1 - Idade 


\begin{tabular}{|c|c|c|c|}
\hline \multicolumn{4}{|c|}{ Tabela II - Fração de encurtamento (\%) } \\
\hline Grupo & A & Grupo B & Grupo C \\
\hline & 14 & 15 & 18 \\
\hline & 12 & 14 & 15 \\
\hline & 16 & 25 & 12 \\
\hline & 16 & 11 & 12 \\
\hline & 19 & 12 & 17 \\
\hline & 13 & & 13 \\
\hline & 10 & & 13 \\
\hline & 10 & & 17 \\
\hline & 11 & & 18 \\
\hline & 5 & & \\
\hline Média & 12,60 & 15,40 & 15,00 \\
\hline DP & 3,95 & 5,59 & 2,55 \\
\hline
\end{tabular}

\begin{tabular}{|c|c|c|c|}
\hline \multicolumn{4}{|c|}{ Tabela III - Fração de ejeção (\%) } \\
\hline \multicolumn{2}{|c|}{ Grupo A } & \multirow{2}{*}{ Grupo B } & \multirow{2}{*}{$\begin{array}{c}\text { Grupo } \mathrm{C} \\
18\end{array}$} \\
\hline & 14 & & \\
\hline & 12 & 20 & 23 \\
\hline & 16 & 15 & 12 \\
\hline & 16 & 21 & 21 \\
\hline & 19 & 23 & 26 \\
\hline & 13 & & 19 \\
\hline & 10 & & 23 \\
\hline & 12 & & 17 \\
\hline & 16 & & 18 \\
\hline \multicolumn{4}{|c|}{8} \\
\hline Média & 13,60 & 18,80 & 19,67 \\
\hline DP & 3,27 & 3,63 & 4,12 \\
\hline
\end{tabular}

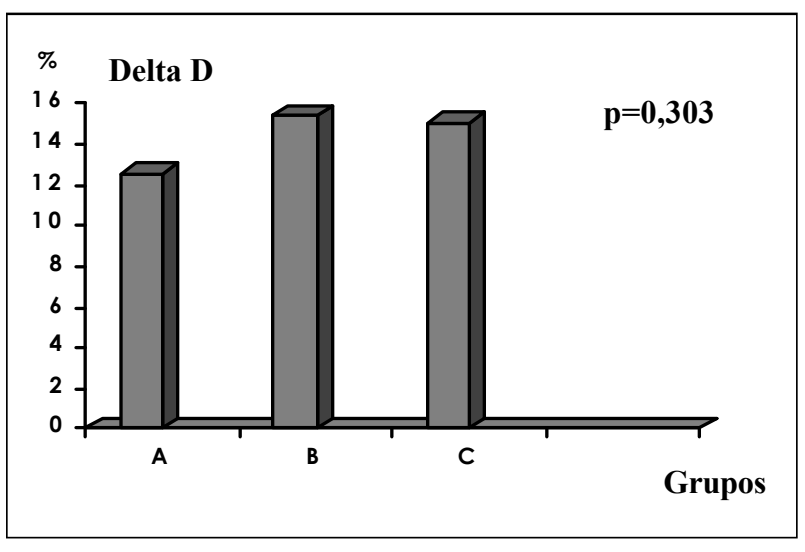

Fig. 2 - Fração de encurtamento (delta D)

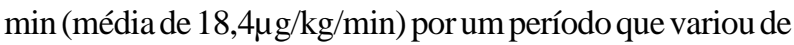
sete a 45 dias (média de 22,6 dias). Em relação aos vasodilatadores, os pacientes do grupo A receberam captopril na dose entre 0,7 a $1,5 \mathrm{mg} / \mathrm{kg} / \mathrm{dia}$ (média de $1,2 \mathrm{mg} / \mathrm{kg} / \mathrm{dia}$ ), no grupo B a dose variou entre 0,5 a $1,25 \mathrm{mg} / \mathrm{kg} /$ dia (média de $0,9 \mathrm{mg} / \mathrm{kg} / \mathrm{dia}$ )e no grupo Cde 0,5 a $1,5 \mathrm{mg} / \mathrm{kg} / \mathrm{dia}$ (média de $1,0 \mathrm{mg} / \mathrm{kg} / \mathrm{dia}$ ).

Em relação à idade, observou-se que a idade média do grupo que recebeu o transplante foi de $35,7 \pm 22,29$ meses, o grupo que aguarda o transplante foi de 27,60 $\pm 12,48$ meses e o grupo que faleceu foi de $64,11 \pm 35,61$ meses. A análise comparativa entre os grupos revelou que houve diferença significativa entre o grupo que faleceu e o grupo que aguarda o transplante ( $\mathrm{p}=0,036)$ (tab. 1 e fig. 1$)$.

No estudo comparativo entre as frações de encurtamento pelo ecocardiograma pôde-se observar que o grupo A que recebeu o transplante foi de $5 \%$ a $19 \%$ média de $12,60 \pm 3,95 \%$, o grupo B apresentou de $11 \%$ a $25 \%$ com média de $15,40 \pm 5,59 \%$, o grupo C variou de $12 \%$ a $18 \%$ com média de $15,00 \pm 2,55 \%$, não havendo diferença estatisticamente significativa entre os grupos $(\mathrm{p}=0,303)(\mathrm{tab}$. II e fig. 2).

$\mathrm{Na}$ análise da ventriculografia radioisotópica, o grupo A apresentou a FEVEentre $8 \%$ e $19 \%$ média de 13,60 $\pm 3,27 \%$, o grupo B entre $15 \%$ e $23 \%$ commédia de $18,80 \pm 3,63 \%$ e o grupo Centre $12 \%$ e $26 \%$, commédiade $19,67 \pm 4,12 \%$, houvediferença significativa entre o grupo A e o grupo $\mathrm{C},(\mathrm{p}=0,004)$ apesar de todos os grupos apresentarem a FE $<26 \%$ (tab. III e fig. 3).

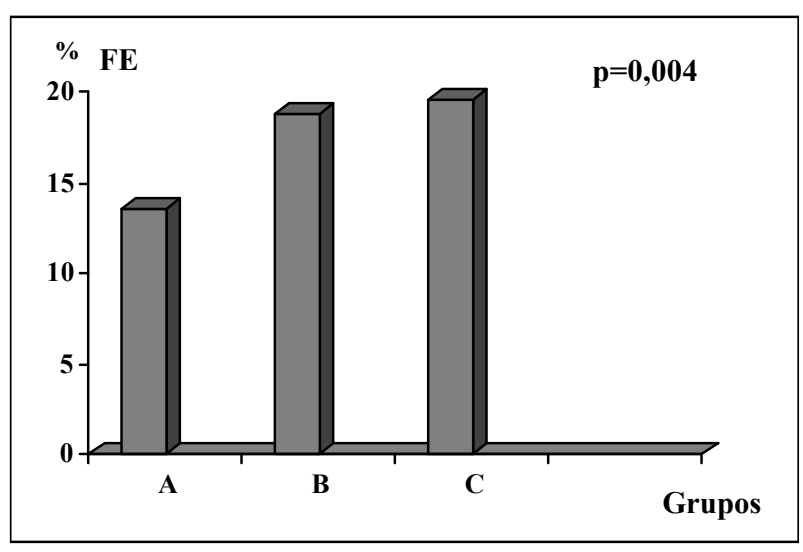

Fig. 3 - Fração de ejeção de ventrículo esquerdo ( FE)

\begin{tabular}{|c|c|c|c|}
\hline \multicolumn{4}{|c|}{ Tabela IV - Débito cardíaco (L/min) } \\
\hline Grupo & A & Grupo B & Grupo C \\
\hline & 1,00 & 2,00 & 1,60 \\
\hline & 3,10 & 2,20 & 1,80 \\
\hline & 1,00 & 1,20 & 2,40 \\
\hline & 1,50 & 2,10 & 2,00 \\
\hline & 1,70 & 2,50 & 1,90 \\
\hline & 1,30 & & 1,80 \\
\hline & 2,20 & & 2,20 \\
\hline & 1,50 & & 1,84 \\
\hline & 1,60 & & 1,70 \\
\hline Média & 1,65 & 2,00 & 1,92 \\
\hline DP & 0,65 & 0,48 & 0,25 \\
\hline
\end{tabular}

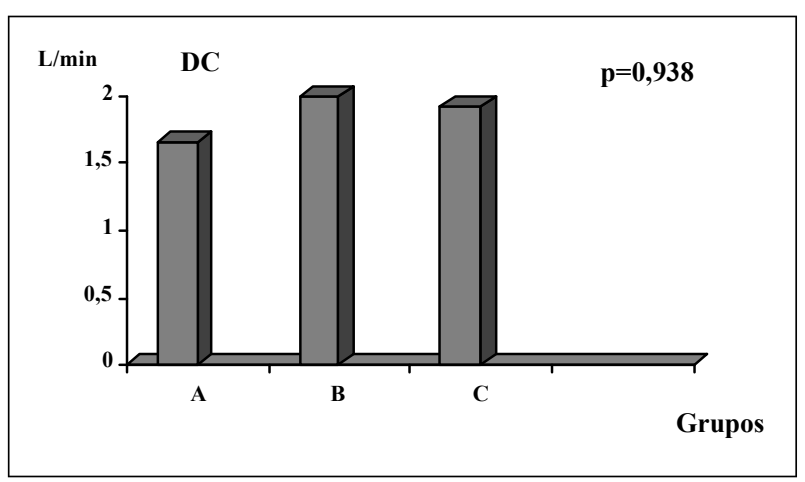

Fig. 4 - Débito cardíaco (DC) 


\begin{tabular}{|c|c|c|c|}
\hline \multicolumn{4}{|c|}{ Tabela V - Pressão média de artéria pulmonar (mmHg) } \\
\hline Grupo & A & Grupo B & Grupo C \\
\hline & 22 & 27 & 37 \\
\hline & 20 & 40 & 28 \\
\hline & 21 & 21 & 45 \\
\hline & 37 & 50 & 49 \\
\hline & 20 & 28 & 50 \\
\hline & 26 & & 45 \\
\hline & 28 & & 30 \\
\hline & 20 & & 58 \\
\hline & 36 & & 34 \\
\hline & 56 & & \\
\hline Média & 28,6 & 33,2 & 41,78 \\
\hline DP & 11,58 & 11,65 & 10,10 \\
\hline
\end{tabular}

\begin{tabular}{|c|c|c|c|}
\hline \multicolumn{4}{|c|}{ Tabela VI - Pressão capilar pulmonar (mmHg) } \\
\hline Grupo & A & Grupo B & Grupo C \\
\hline & 4 & 17 & 22 \\
\hline & 15 & 25 & 16 \\
\hline & 15 & 13 & 35 \\
\hline & 27 & 15 & 13 \\
\hline & 12 & 14 & 31 \\
\hline & 11 & & 17 \\
\hline & 18 & & 10 \\
\hline & 12 & & 35 \\
\hline & 29 & & 2 \\
\hline & 25 & & \\
\hline Média & 17,8 & 16,80 & 20,11 \\
\hline DP & 6,71 & 4,82 & 11,58 \\
\hline
\end{tabular}

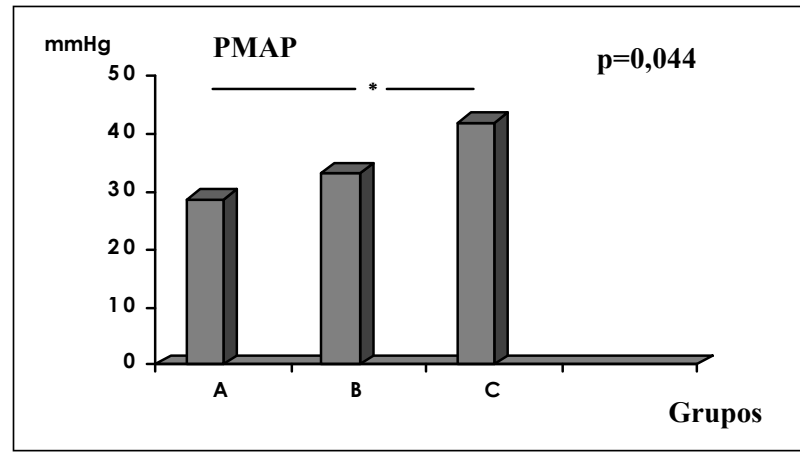

Fig. 5 - Pressão média de artéria pulmonar (PMAP)

No estudo hemodinâmico observou-se que o débito cardíaco variou de 1 a 3,1L/min com média de $1,65 \pm 0,65 \mathrm{~L} /$ min, no grupo A, no grupo B de 1,2 a 2,5L/min com média de $2,0 \pm 0,48 \mathrm{~L} / \mathrm{min}$ e no grupo Centre $1,6 \mathrm{e} 2,4 \mathrm{~L} / \mathrm{min}$ com média de $1,92 \pm 0,25 \mathrm{~L} / \mathrm{min}$. Não houve diferença estatística entre os grupos (tab. IV e fig. 4).

APMAPno grupo A variou de 20 a $56 \mathrm{mmHg}$ com média de $28,60 \pm 11,58 \mathrm{mmHg}$, no grupo $B$ variou de 21 a $50 \mathrm{mmHg}$ com média de $33,20 \pm 11,65 \mathrm{mmHg}$ e no grupo C de 28 a $58 \mathrm{mmHg}$ com média de $41,78 \pm 10,10 \mathrm{mmHg}$. Houve diferença estatística entre o grupo A e o grupo $\mathrm{C}(\mathrm{p}=0,044)$ (tab. V e fig. 5).

Em relação à pressão capilar média, o grupo que recebeu o transplante apresentou pressão entre 11 e $29 \mathrm{mmHg}$ com média de $17,8 \pm 6,71 \mathrm{mmHg}$, a pressão no grupo $B$ foi de 13 a $25 \mathrm{mmHg}$ com média de $16,80 \pm 4,82 \mathrm{mmHg}$, o grupo de pacientes que faleceram apresentou variação entre $2 \mathrm{e}$ $35 \mathrm{mmHg}$ com média de $20,11 \pm 11,58 \mathrm{mmHg}$. Não houve diferença significativa entre os grupos analisados $(\mathrm{p}=0,508)$ (tab. VIefig. 6).

O IRVP no grupo que recebeu o transplante apresentou entre 1,00 e 7,10 Wood com média de 3,44 $\pm 1,95$ Wood, no grupo que aguarda o transplante (B) o índice variou entre 2,20 e 8,80 Wood com média de 5,15 $\pm 2,71$ Wood, o grupo C variou entre 4,00 e 12,50 Wood com média de $8,41 \pm 4,24$ Wood. Houve diferença significativa entre os grupos $\mathrm{A}$ e $\mathrm{C}(\mathrm{p}=0,010)$ (tab. VII e fig. 7). Os pacientes com IRVP $>6$ Wood aguardavam transplante cardíaco heterotópico.

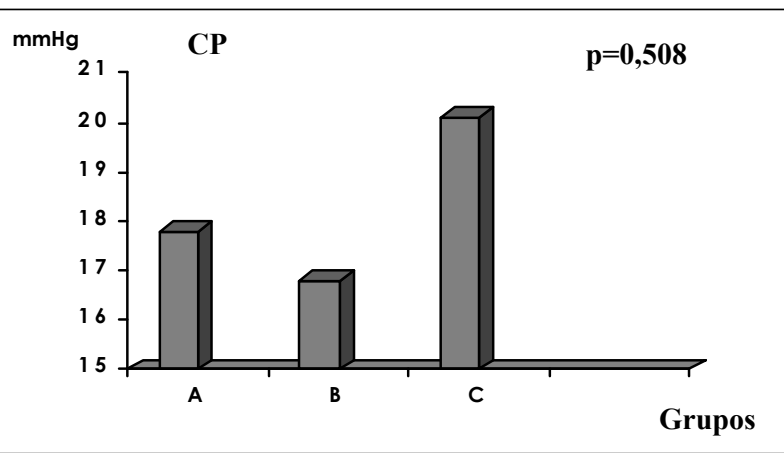

Fig. 6 - Pressão capilar pulmonar (CP)

\begin{tabular}{|ccc|}
\hline \multicolumn{5}{|c|}{ Tabela VII - Índice de resistência vascular pulmonar } \\
(unidades Woods)
\end{tabular}

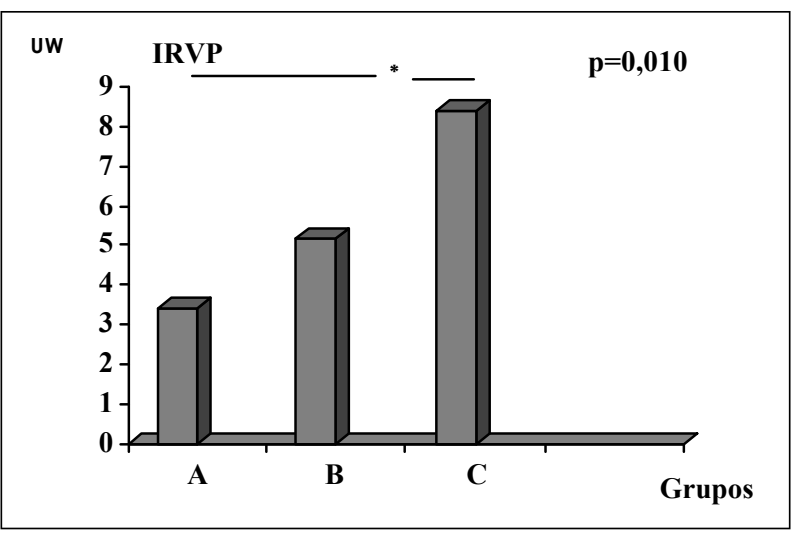

Fig. 7 - Índice de resistência vascular pulmonar (IRVP) 


\begin{tabular}{|c|c|c|}
\hline \multicolumn{3}{|c|}{ Tabela VIII - Gradiente de pressão transpulmonar (mmHg) } \\
\hline Grupo A & Grupo B & Grupo C \\
\hline 8 & 10 & 15 \\
\hline 5 & 15 & 12 \\
\hline 6 & 8 & 10 \\
\hline 10 & 35 & 36 \\
\hline 8 & 14 & 19 \\
\hline 15 & & 28 \\
\hline 10 & & 20 \\
\hline 8 & & 23 \\
\hline 7 & & 32 \\
\hline 31 & & \\
\hline Média 10,8 & 16,40 & 21,67 \\
\hline DP $\quad 7,61$ & 10,78 & 8,93 \\
\hline
\end{tabular}

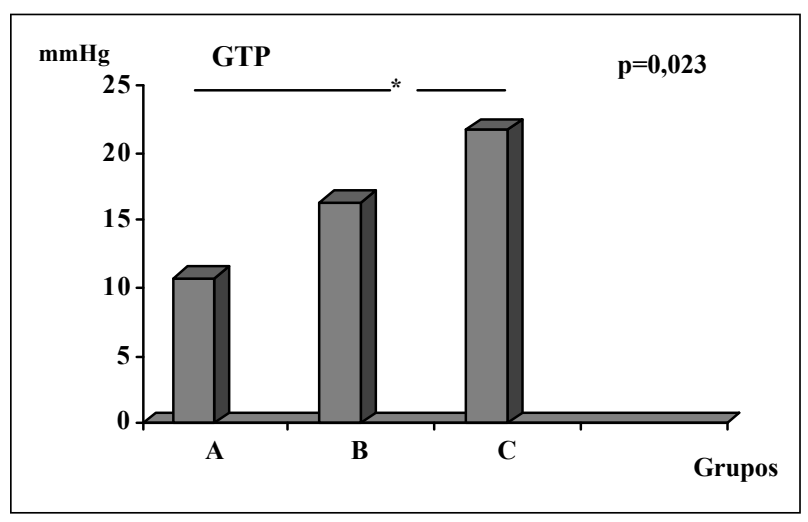

Fig. 8 - Gradiente de pressão transpulmonar (GTP)

Em relação ao GPT pôde-se observar que o grupo A apresentou ogradienteentre $5 \mathrm{e} 31 \mathrm{mmHg}$ médiade $10,8 \pm 7,61 \mathrm{mmHg}$. No grupo $\mathrm{B}$, o gradiente variou de 8 a $35 \mathrm{mmHg}$, com média de $16,4 \pm 10,78 \mathrm{mmHg}$ e no grupo Centre 10 e $36 \mathrm{mmHg}$ com média de 21,67 $\pm 8,93 \mathrm{mmHg}$. Houve diferença significativa entre os grupos $\mathrm{Ae} \mathrm{C}(\mathrm{p}=0,023)$ (tab. VIII e fig. 8).

\section{Discussão}

O quadro clínico e a fisiopatologia da cardiomiopatia dilatada são bem conhecidos. A característica clássica é a disfunção ventricular de ambos os ventrículos, embora um possa ser dominante, levando a sinais congestivos de IC. Benson em $1991{ }^{15}$ relata que a incidência de cardiomiopatia na população pediátrica é cerca de $0,94 \%$ do total de pacientes pediátricos que foram avaliados no Hospital Infantil Sick Children de Toronto, nos últimos 30 anos, tendo como causa a fibroelastose endomiocárdica. A incidência anual de cardiomiopatia baseada na população adulta varia de 7 a 7,5 casos por 100.000 , sendo que mais de $0,7 \%$ de óbitos cardiológicos têm sido atribuídos à cardiomiopatia. Acredita-se que cardiomiopatia dilatada constitui mais de $90 \%$ de todas as cardiomiopatias, principalmente na população pediátrica. Benson ${ }^{15}$ relata ainda que a história natural da cardiomiopatia dilatada é função da precocidade do diagnóstico da doença. A mortalidade de um ano é de $23 \%$ e de dois anos é de $48 \%$ em adultos com IC grave.
Há poucos trabalhos da evolução natural da cardiomiopatia dilatada em crianças. Greenwood e $\mathrm{col}^{3}$ relatam que de 161 pacientes pediátricos com vários graus de processos miocárdicos incluindo miocardites, apresentaram mortalidade de $35 \%$, sendo que $56 \%$ morreram no $1^{\circ}$ mês de diagnóstico e $77 \%$ no $1^{\circ}$ ano de evolução. Observaram também que aproximadamente um terço dos casos acaba falecendo, um terço dos pacientes melhora e um terço das crianças passa para a cronicidade. Unverferth e $\mathrm{col}^{4}$, em estudo realizado na população adulta de fatores que influenciam a mortalidade em um ano, revelaram que os mais importantes foram arritmias ventriculares e pressão média de átrio direito.

Taliercio e $\mathrm{col}^{16}$, em estudo realizado na Clínica Mayo, relatam mortalidade de $66 \%$ em cinco anos de 24 pacientes pediátricos. Não foram observados fatores preditivos de evolução, embora tenha-se concluído que o prognóstico da cardiomiopatia dilatada em crianças é freqüentemente grave, e que a minoria mostra evolução clínica imprevisível. A idade no momento do diagnóstico influencia a história natural e pacientes com idade $<2$ anos (principalmente os portadores de endomiocardiofibrose) parecem ter pior prognóstico, embora esta evolução não seja observada em todos os centros.

Griffin e $\mathrm{col}^{17}$, em estudo realizado em 20 pacientes $<2$ anos de idade e 12 crianças $>2$ anos, observaram que 5 $(25 \%)$ do $1^{\circ}$ grupo faleceram mostrando fibroelastose em quatro dos pacientes. Todos os pacientes com idade $>2$ anos faleceram. O grupo que faleceu revelou diferença significativa em relação à incidência de história familiar, distúrbios de ritmo cardíaco. Observaram também como fatores de risco, cardiomegalia persistente e desenvolvimento de arritmias significativas, concluindo que o transplante cardíaco está indicado em crianças $>2$ anos de idade que sobrevivem mais de um mês após o diagnóstico, crianças $<2$ anos cujo quadro não melhora após um ano e que tenham cardiomegalia persistente ou arritmias ventriculares complexas. Nopresente estudo a idade mostrou diferença significativa entre $o$ grupo que faleceu e o grupo que aguarda o transplante.

Chen Su e col ${ }^{6}$ estudaram 23 crianças comparando o grupo que sobreviveu (12) e o grupo que faleceu (11) em relação à idade de início dos sintomas, sexo, índice cardiotorácico pela radiografia de tórax, ECG com padrão de infarto do miocárdico, alterações de ST-T ou arritmias e pressão de enchimento diastólica de ventrículo esquerdo (VE). Não observaram diferença significativa desses parâmetros entre os dois grupos, entretanto a fração de encurtamento entre o grupo que faleceu (média de 11,5\%) em relação ao de 20,9\% no grupo que sobreviveu foi significativa, $\mathrm{p}<0,01$, além de história familiar e endomiocardiofibrose que indicam mau prognóstico. Neste trabalho a média da fração de encurtamento foi de $15,14 \% \pm 5,59 \mathrm{e} 15,00 \% \pm 2,55$, mostrando a gravidade em ambos os grupos $\mathrm{B}$ e C; não observamos diferença entre os mesmos.

Friedman e $\mathrm{col}^{5}$, em estudo retrospectivo de 63 crianças portadoras de cardiomiopatia dilatada com média de idades de 4,96 $\pm 5,3$ anos, constataram uma mortalidade de $16 \%$, observando que IC persistente e alterações no segmento ST-T correlacionaram-se com aumento de mortalidade $(\mathrm{p}<0,05)$. 
Ciszewski e col ${ }^{18}$ estudaram a evolução de 19 portadores de cardiomiopatia dilatada com CF variável de I IV e idades entre dois e 18 (média de 13,4 \pm 4 ) anos sendo que 12 pacientes sobreviveram e sete faleceram. Não observaram diferença significativa entre os dois grupos em relação aos seguintes parâmetros: incidência de IC e arritmias graves, fração de encurtamento e parâmetros hemodinâmicos como índice cardíaco, pressão diastólica de VE e índice de volume diastólico de VE. No nosso estudo, do ponto de vista hemodinâmico, não observamos diferença entre os grupos B e C. No entanto, o IRVP, PMAP e o GPT mostraram diferenças significativas entre os grupos $\mathrm{A} \mathrm{e} \mathrm{C}$, embora todos os nossos pacientes estivessem em CF IV. Apesar dos elevados IRVP estarem mais freqüentemente associados às crianças portadoras de cardiopatias congênitas, estes dados nos mostram que as portadoras de cardiomiopatia dilatada também podem apresentar esta complicação.

Os inotrópicos, vasodilatadores e diuréticos e atualmente os betabloqueadores são drogas que devem ser utilizadas o mais precoce possível antes da indicação do transplante e mantidas mesmo após sua indicação.

O transplante cardíaco pediátrico é considerado atualmente a opção terapêutica de escolha em crianças portadoras de cardiopatias congênitas complexas e de cardiomiopatias refratárias à terapêutica convencional. Embora este procedimento esteja consagrado, o melhor momento de sua indicação é aquele onde a criança receptora tenha condi- ções hemodinâmicas mínimas de aguardar o transplante e que obtenha benefícios com o procedimento, uma vez que o número de potenciais doadores é escasso e a maioria falece enquanto aguarda o órgão compatível.

McGiffin e col ${ }^{19}$ relataram que do total de 264 pacientes entre três dias e 17,9 anos de idade, média de 4,7 anos, listados no Grupo de Estudos de Transplante Cardíaco Pediátrico nos Estados Unidos, incluindo cardiopatias congênitas e adquiridas, $158(60 \%)$ foram transplantados, $60(23 \%)$ faleceram e $36(14 \%)$ aguardam. No nosso trabalho, $41,6 \%$ das crianças receberam o transplante, $20,8 \%$ aguardam na lista, enquanto $37,6 \%$ faleceram. O maior número de óbitos no nosso estudo pode ser conseqüência da pior condição hemodinâmica no momento de indicação do transplante e da escassez de doadores no nosso meio (número absoluto, compatibilidade entre receptor e doador quanto à tipagem sangüínea, peso, cross-match, etc).

Em conclusão, observamos que o perfil dos parâmetros hemodinâmicos das crianças portadoras de cardiomiopatia dilatada grave foi compatível com o quadro clinico de IC refratária. A idade foi o fator significativo que diferenciou o grupo B do C. OIRVP, PMAP e o GPT foram fatores que diferenciaram de modo significativo o grupo que faleceu (grupo C) e o de pacientes transplantados (grupo A). A média do IRVP nos pacientes que aguardam o transplante apresentou um valor intermediário entre os outros dois grupos A e C. Quanto maior a idade da criança no momento de indicação do transplante, pior o seu prognóstico.

\section{Referências}

1. Camargo PR, Snitcowsky R, Mazzieri R et al - Favorable effects of immunosupressive therapy in children with dilated cardiomyopathy and active myocarditis. Pediatric Cardiol 1995; 16: 61-8.

2. Taliercio CP, Driscoll DJ, Seward JB et al - Outcome of 33 children with dilated cardiomyopathy: Importance of clinical status at 1 year. Supplement II Circulation, 1989; 4: II-282

3. Greenwood R, Nadas AS, Fyler DC - The clinical course of primary myocardial disease in infants and children. Am Heart J 1976; 5: 549-60.

4. Unverferth DV, Magorien RD, Moeschberger ML et al - Factors influencing the one-year mortality of dilated cardiomyopathy. Am J Cardiol 1984; 54: 147-52.

5. Friedman RA, Moak JP, Garson A - Clinical course of idiopathic dilated cardiomyopathy in children. J Am Coll Cardiol 1991; 18: 152-6.

6. Chen S, Nouri S, Balfour I et al - Clinical profile of congestive cardiomyopathy in children. J Am Coll Cardiol 1990; 15: 189-93.

7. Addonizio LJ, Hsu DT, Fuzesi L et al - Optimal timing of pediatric heart transplantation. Circulation 1989; 80(suppl III): III-84-9.

8. Addonizio LJ - Cardiac transplantation in the pediatric patient. Dilated cardiomyopathy in infants and children. Progr Cardiovasc Dis 1990; 1: 19-34.

9. Barbero Marcial M, Azeka E, Camargo PR et al - Transplante cardíaco neonatal e infantil. Arq Bras Cardiol 1996; 67: 165-70.

10. Kirklin JK, Naftel DC, McGiffin DC et al - Analysis of morbid events and risk factors for death after cardiac transplantation. J Am Coll Cardiol 1988; 11: 917-24.
11. Kawaguchi A, Gandjbakhch I, Pavies A et al - Cardiac transplant recipients with preoperative pulmonary hypertension. Circulation 1989; 80(suppl III): III-90-6.

12. Addonizio LJ, Gersony WM, Robbins RC et al - Elevated pulmonary vascular resistance and cardiac transplantation. Circulation 1987; 76: V-52.

13. Houde C, Desmond JB, Freedom RM et al - Profile of pediatric patients with pulmonary hypertension judged by responsiveness to vasodilators. Br Heart J 1993; 70: 461-8

14. Jackle AC, Fowler MB - Influence of preoperative pulmonary artery pressure on mortality after heart transplantation: testing of potencial reversibility of pulmonary hypertension with nitroprusside is useful in defining a high risk group. J Am Coll Cardiol 1992; 19: 48-54.

15. Benson L-The failing heart - myopathies. J Heart Lung Transplant 1991; 10: 793- 5 .

16. Taliercio CP, Seward JB, Driscoll DJ et al - Idiopathic dilated cardiomyopathy in the young: Clinical profile and natural history. J Am Coll Cardiol 1985; 6: 1126-31.

17. Griffin ML, Hernandez A, Martin TC et al - Dilated cardiomyopathy in infants and children. J Am Coll Cardiol; 1988; 11: 139-44.

18. Ciszewski A, Bilinska T, Lubiszewska B et al - Dilated cardiomyopathy in children: Clinical course and prognosis. Pediatric Cardiol 1994; 15: 121-6.

19. McGiffin DC, Naftel DC, Kirklin JK et al - Predicting outcome after listing for heart transplantation in children: comparison of Kaplan-Meier and parametric competing risk analysis. J Heart Lung Transplant 1997; 16: 713-22. 